\title{
Telehealth: The role of respiratory therapists during the COVID-19 emergency
}

\author{
Mrudula M. Sawadkar M.Sc RT ${ }^{1}$, Varun R Nayak M.Sc RT ${ }^{1}$
}

MM Sawadkar, VR Nayak. Telehealth: The role of respiratory therapists during the COVID-19 emergency. Can J Respir Ther 2021;57:119-120. doi: 10.29390/cjrt-2021-039.

Key Words: Telehealth; telerehabilitation; respiratory therapist; respiratory care; COVID-19 pandemic

The COVID-19 pandemic has emerged as a deadly disease worldwide. The second wave is a super spreader in India by taking the overall COVID-19 infection toll to 25 million with total mortality of 2,80,000 [1]. The surge in symptomatic patients increased the requirement of medical facilities including intensive care unit (ICU) beds, oxygen requirements, medications, and number of health care professionals (HCPs) for treatment of these patients [2]. The respiratory therapists (RTs) are working tirelessly with all other major health care entities in tackling this acute emergency [3]. Even though the number of RTs working in India is marginal, we still have a very high scope of serving patients during this acute pandemic emergency. Currently, the majority of the Indian RTs are working in the ICUs and COVID units of the hospital. Apart from the regular hospital duties, an RT can help the patients and the different primary health care centers using telehealth.

Telehealth uses electronic communication for transferring medical information from one area to another for optimizing health care services [4]. It uses information technology in the form of email chats, telephone calls, video calls, and application-based monitoring for the delivery of health care services $[4,5]$. The developed countries have been using these telehealth services for a long time and optimized their use during the COVID-19 pandemic. But, for developing countries like India, the use of telehealth is still a difficult task because of the lack of resources and the knowledge to utilize the technology. As India is facing the second wave of COVID-19 disease with a very high positivity rate and mortality, the situation has pushed the medical setup to utilize telehealth for the delivery of health care services. Telehealth services are utilized in various COVID/non-COVID patients for routine assessments, monitoring, and management of the patients. The majority of the COVID patients will present with mild to moderate symptoms for whom home-based telehealth monitoring will be helpful.

There are certainly three major aspects an RT can help as a telehealth provider during the current pandemic (Figure 1). COVID patients with pulmonary comorbidities require frequent tele-monitoring and care during the pandemic. For the COVID patients, an RT can note the routine pulmonary-specific symptoms and vital signs through video calling. Routine monitoring of the disease progression and alerting the patient as well as the hospital for the requirement of medical attention are the important factors for saving the patient's life. Apart from assessment and monitoring, an RT can educate the patient on the use of pulse oximetry, symptom management, use of breathing exercises, awake proning, and the use of oxygen therapy devices including cylinders and oxygen concentrators and aerosol therapy devices.

Patients who require pulmonary rehabilitation are affected during the COVID 19 pandemic worldwide [6]. The nationwide lockdown and lack of availability of transportation to seek medical attention, increased COVID patient load in the tertiary care hospitals, reduced outpatient department hours, and the general fear of COVID-19 infection in these patients have affected their quality of life. An RT can assist them through tele-rehabilitation, thereby improving the quality of life during this unforeseen situation. Through telerehabilitation, we can assess the patient, note daily symptom progression, provide psychological support, and educate them in using various breathing exercises and respiratory care equipment they use for daily respiratory support [6]. Self-management plays an important role in patients who require pulmonary rehabilitation [7]. An application-based patient monitoring and video monitored telerehabilitation would reduce the frequent exacerbations and improve the quality of life in these patients.

The third important aspect where RTs can help is strengthening the respiratory care functioning in primary health care centers and nursing homes. The acute rise in COVID cases warrants the requirement of all nursing care units and primary health centers for acute patient management. We can educate the health care providers working in these facilities on the use of oxygen cylinders and oxygen concentrators, the use of various oxygen therapy devices, use of home care and transport ventilators, and other emergency cardiorespiratory management that is necessary for patient survival. These education programs can be easily adapted using the telehealth platform.

In conclusion, The COVID-19 pandemic has put the health care system into an emergency. The surge in COVID patients during the second wave in India showed the lack of resources to manage the patients and put the health care system into crisis. During these situations of demand, the country and the government should look into utilizing the working RTs in various telehealth services to provide quality care for the patients. The RTs can provide quality care for the COVID patient who is under quarantine, patients who are seeking rehabilitation, and educate the health care providers who are working in the primary health care centers on various respiratory care services.

\footnotetext{
${ }^{1}$ Department of Respiratory Therapy, Manipal College of Health Professions, Manipal Academy of Higher Education, Karnataka, India

Correspondence: Varun R Nayak, Department of Respiratory Therapy, Manipal College of Health Professions, Manipal Academy of Higher Education, Manipal, Karnataka-576104, India, Tel: +91 9972381165, E-mail: Varunr838@gmail.com
} 


\section{FIGURE 1.}

Roles of respiratory therapist during the COVID-19 emergency. $\mathrm{QOL}=$ quality of life; $\mathrm{HCP}=$ health care professionals.

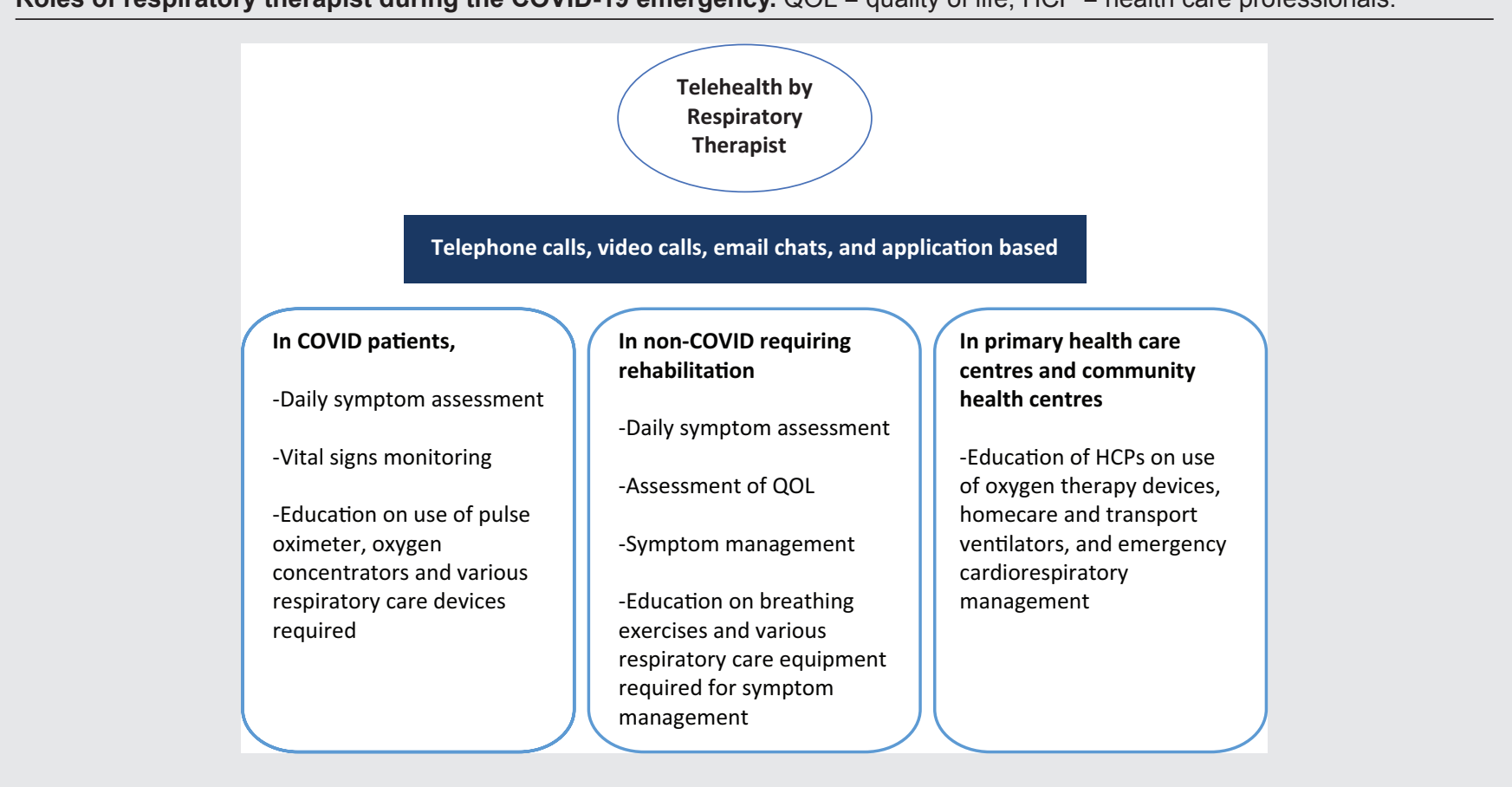

\section{REFERENCES}

1. World Health Organization. WHO Health Emergency Dashboard. Available at: https://covid19.who.int/region/searo/country/in

2. Jain VK, lyengar KP, Vaishya R. Differences between first wave and second wave of COVID-19 in India. Diabetes Metab Syndr. 2021;15(3): 104748. doi: 10.1016/j.dsx.2021.05.009

3. Sawadkar MM, Nayak VR. Respiratory therapists: the unnoticed warriors during COVID-19 pandemic in India. Can J Respir Ther 2020;56:57. doi: 10.29390/cjrt-2020-044

4. Jnr BA. Use of telemedicine and virtual care for remote treatment in response to COVID-19 pandemic. J Med Syst 2020;44(7):1-9. doi: $10.1007 / \mathrm{s} 10916-020-01596-5$
5. Bokolo AJ. Application of telemedicine and eHealth technology for clinical services in response to COVID-19 pandemic. Health Technol 2021;11(2):359-66. doi: 10.1007/s12553-020-00516-4

6. Rutkowski, S. Management challenges in chronic obstructive pulmonary disease in the COVID-19 pandemic: telehealth and virtual reality. J Clin Med 2021;10:1261. doi: 10.3390/jcm 10061261

7. Hill K, Vogiatzis I, Burtin C. The importance of components of pulmonary rehabilitation, other than exercise training, in COPD. Eur Respir Rev 2013;22(129):405-13. doi: 10.1183/09059180. 00002913 